\title{
Measurements of Higgs boson production in decays to two tau leptons with the ATLAS detector
}

\section{David Hohn*, on behalf of the ATLAS Collaboration}

Physikalisches Institut, Albert-Ludwigs-Universität Freiburg, Germany

E-mail: david.hohn@eern.ch

\begin{abstract}
Measurements of cross sections for the production of Higgs bosons decaying to tau lepton pairs performed by the ATLAS experiment, using LHC proton-proton collision data from 2015 and 2016 at a centre of mass energy of $13 \mathrm{TeV}$, are presented. All measured values are consistent with their Standard Model (SM) predictions. Additionally it is possible to probe CP properties with these event signatures. A search for $\mathrm{CP}$ violation in the production vertex of the Higgs boson via vector boson fusion production at $8 \mathrm{TeV}$ finds no deviations from the SM and sets limits on a CP-mixing parameter in the $H V V$ vertex at $68 \%$ confidence level (CL). The High Luminosity LHC will allow the CP-mixing in the Higgs boson decay to tau leptons pairs to be probed, and to set limits at $95 \%$ CL.
\end{abstract}

European Physical Society Conference on High Energy Physics - EPS-HEP2019 -

10-17 July, 2019

Ghent, Belgium

${ }^{*}$ Speaker. 


\section{Introduction}

Measuring the properties of the Higgs boson is an important part of the mission of the ATLAS experiment [1] at the LHC ever since the boson's discovery was announced in 2012. Of particular interest are Higgs boson processes that involve the decay to a pair of tau leptons.

There are two major reasons for this. One is the fact that interactions between the Higgs boson and fermions (such as the tau lepton) are so-called Yukawa interactions. This means that they do not arise from considerations of (local) gauge invariance and are therefore unlike any other known interactions between elementary particles. It is thus of particular interest to determine whether they behave consistently with what is predicted by the mechanism of spontaneous symmetry-breaking [2-4] and additional scalar-fermion Yukawa interactions [5]. The other reason is that the decay of the Higgs boson to a pair of tau leptons represents a large and detectable fraction of all Higgs boson decays. As such it provides large sample sizes of Higgs boson events. Likewise, production modes with small cross sections such as gluon-gluon fusion (ggF) where the Higgs boson has large transverse momentum and vector boson fusion (VBF) are accessible for measurement.

Additionally it is possible to probe $\mathrm{CP}$ properties of the Higgs boson by exploiting the presence of spin correlations both in the production vertex $H V V$ in VBF and the decay vertex $H f f$.

This work summarises recent measurements of Higgs boson production cross sections at $\sqrt{s}=$ $13 \mathrm{TeV}$ [6], a test for $\mathrm{CP}$ invariance in VBF production of the Higgs boson at $\sqrt{s}=8 \mathrm{TeV}$ [7] and prospects for measuring the $\mathrm{CP}$ nature of the Higgs-boson-tau-lepton coupling at the high luminosity upgrade to the LHC (HL-LHC) [8].

\section{Signatures of a Higgs boson decay to pairs of tau leptons}

Tau leptons decay weakly to the less massive charged leptons, like $\tau \rightarrow \ell v_{\ell} v_{\tau}$, where $\ell$ signifies electron or muon. These are called leptonic tau decays or $\tau_{\text {lep }}$, and are efficiently reconstructed and identified as electrons or muons.

Tau leptons also decay to hadrons, most commonly to 1 or 3 charged pions and optionally additional neutral pions. These are called hadronic tau decays or $\tau_{\text {had }}$, and are reconstructed as jets. Discrimination of tau jets from jets initiated by quarks or gluons is achieved by a multivariate discriminant that considers secondary vertex, track impact parameter and shower shape observables. To reduce the large number of background hadronic jets that may be misidentified as tau jets, the critical value for the identification discriminant is chosen with a low efficiency to identify true taus of $40-55 \%$ depending on the number of charged hadrons in the tau lepton decay [9].

Higgs boson decays to pairs of tau leptons are thus categorised according to the characteristic tau lepton decays by selecting all combinations of two tau lepton decays, into $\tau_{\text {lep }} \tau_{\text {lep }}, \tau_{\text {lep }} \tau_{\text {had }}$ and $\tau_{\text {had }} \tau_{\text {had }}$, where in each there is a trade-off between branching ratio, background contamination and reconstructed energy resolution.

Only the visible part of the tau lepton decay, without neutrinos, is reconstructed in both $\tau_{\text {lep }}$ and $\tau_{\text {had }}$. To recover the invisible neutrino information ATLAS employs the missing mass calculator (MMC) [10]. The MMC assumes that the missing transverse momentum is caused by the neutrinos of a resonant ditau decay. It estimates the 4-momenta of the visible and invisible tau lep- 
ton decay products and thereby also the mass of the decaying resonance by maximising a kinematic likelihood. Figure 1 shows that the MMC mass observable provides good discrimination between $H \rightarrow \tau \tau$ and $Z \rightarrow \tau \tau$ decays.

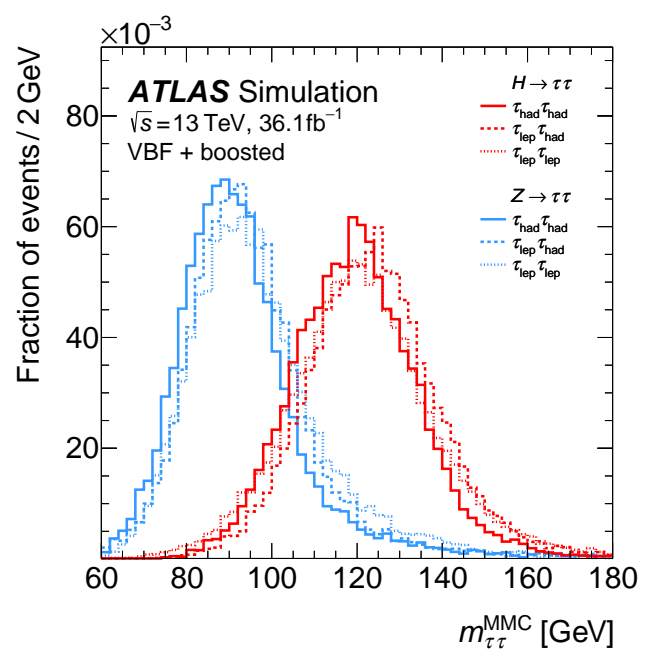

Figure 1: The MMC mass distribution for the $H \rightarrow \tau \tau$ and $Z \rightarrow \tau \tau$ processes split by analysis category used in the cross section measurement at $13 \mathrm{TeV}$ [6].

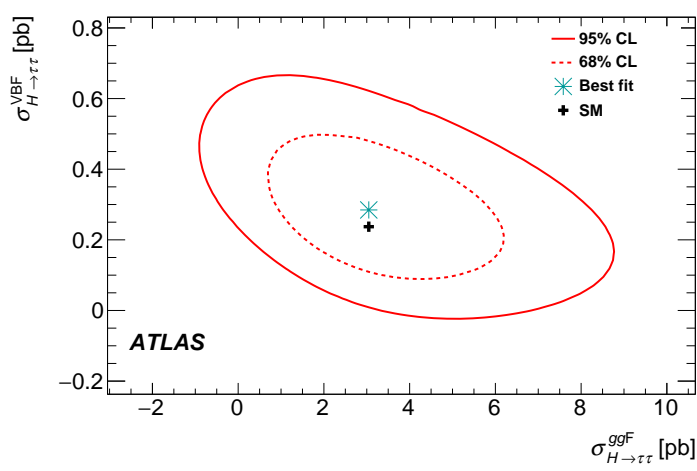

Figure 2: Likelihood contours for the measured cross sections at $13 \mathrm{TeV}$ in the $\left(\sigma_{H \rightarrow \tau \tau}^{\mathrm{ggF}}, \sigma_{H \rightarrow \tau \tau}^{\mathrm{VBF}}\right)$ plane. The $68 \%$ and $95 \% \mathrm{CL}$ contours are shown as dashed and solid lines, respectively, for $m_{H}=125 \mathrm{GeV}$. The SM expectation is indicated by a plus symbol and the best fit to the data is shown as a star [6].

\section{Measurement of cross sections}

A measurement of the cross section of the $H \rightarrow \tau \tau$ process is performed with data recorded by the ATLAS detector corresponding to an integrated luminosity of $36.1 \mathrm{fb}^{-1}$ of proton-proton collisions at a centre-of-mass energy of $13 \mathrm{TeV}$ at the LHC [6].

Events are selected for the presence of exactly two reconstructed tau leptons, in all combinations of leptonic and hadronic decays. To enhance the sensitivity to VBF production, selection requirements are added. Two additional jets must have a large invariant mass, large pseudorapidity separation and be in opposite hemispheres. The Higgs boson decay products are central, in between the two jets. For events that are not classified to be consistent with VBF, further requirements select a large reconstructed Higgs boson transverse momentum, which enhance the sensitivity to the ggF production mode with additional jets and a large Higgs boson boost (denoted "Boosted").

The dominant backgrounds are $Z \rightarrow \tau \tau$ and misidentified hadronically decaying tau leptons in $W+$ jets and multijet events. The $Z \rightarrow \tau \tau$ background is estimated by event samples generated by Monte Carlo simulation techniques. The validity of the simulation is extensively validated using $Z \rightarrow e e / \mu \mu$ events in a phase space that is enriched with those events. Background from misidentified tau leptons is estimated with data-driven techniques.

The observable used for discriminating the $H \rightarrow \tau \tau$ signal from background is the Higgs boson candidate mass, reconstructed with the MMC. The statistical interpretation of the results consists of a binned profile likelihood fit. The setup of the fit model is summarised in Table 1. In total there are 
95 bins. The fit estimates several parameters, which are either completely or partially free. There are 1-3 completely free parameters, representing normalisation of signal. For the backgrounds there are 2 for $Z \rightarrow e e / \mu \mu, 2$ for $Z \rightarrow \tau \tau, 4$ for top-quark, and 1 for processes with misidentified tau leptons.

Systematic uncertainties that affect the result are due to detector performance, background estimation techniques, the limited size of simulated samples as well as theoretical predictions. These are taken into account by the partially free parameters that are also estimated in the fit. They are only partially free as they come with a most likely value which is measured in auxiliary measurements. The most important contributions to the uncertainty are the predictions for the ggF process with selection requirements that exclusively select the number of additional jets, the limited size of MC samples and jet calibration uncertainties that affect the discriminating power of the MMC mass observable.

\begin{tabular}{|c|c|c|c|c|c|c|}
\hline \multirow{2}{*}{$\begin{array}{l}\text { Category } \\
\text { Subcategory }\end{array}$} & \multicolumn{2}{|c|}{$\tau_{\text {lep }} \tau_{\text {lep }}$} & \multicolumn{2}{|c|}{$\tau_{\text {lep }} \tau_{\text {had }}$} & \multicolumn{2}{|c|}{$\tau_{\text {had }} \tau_{\text {had }}$} \\
\hline & VBF & Boosted & VBF & Boosted & VBF & Boosted \\
\hline Number of bins & $7+5$ & $8+7$ & $6+6$ & $6+6$ & $6+6+6$ & $10+10$ \\
\hline $\begin{array}{l}\text { Control regions } \\
\text { ( } 1 \text { bin each) }\end{array}$ & 2 & 2 & 1 & 1 & - & - \\
\hline
\end{tabular}

Table 1: Summary of fit model in the cross section measurement at $13 \mathrm{TeV}$. The discriminant observable is the MMC mass in all categories. A plus (+) sign signifies further categorisation within the indicated categories. Information extracted from [6].

The result of the analysis is strong evidence of the $H \rightarrow \tau \tau$ process with a significance of 4.4 standard deviations (expected 4.1) over the background from other SM processes. In combination with results obtained by analysing 7 and $8 \mathrm{TeV}$ data the significance is 6.4 (expected 5.4) standard deviations. The total measured cross section at $13 \mathrm{TeV}$ is $\sigma_{H \rightarrow \tau \tau}=3.77_{-0.59}^{+0.60}$ (stat. $)_{-0.74}^{+0.87}$ (syst.) pb which is consistent with the prediction of $\sigma_{H \rightarrow \tau \tau}^{\mathrm{SM}}=3.46 \pm 0.13 \mathrm{pb}$.

Additionally, the cross sections is extracted for the ggF and VBF processes separately to be $\sigma_{H \rightarrow \tau \tau}^{\mathrm{ggF}}=3.1 \pm$ (stat.) $)_{-1.3}^{+1.6}$ (syst.) pb and $\sigma_{H \rightarrow \tau \tau}^{\mathrm{VBF}}=0.28 \pm 0.09$ (stat.) $)_{-0.09}^{+0.11}$ (syst.) pb which agree with the SM predictions of $\sigma_{\mathrm{ggF}, H \rightarrow \tau \tau}^{\mathrm{SM}}=3.05 \pm 0.13 \mathrm{pb}$ and $\sigma_{\mathrm{VBF}, H \rightarrow \tau \tau}^{\mathrm{SM}}=0.237 \pm 0.006 \mathrm{pb}$. The $68 \%$ and $95 \%$ confidence contours are shown in Figure 2.

Furthermore, it is possible to split the ggF process into low and high boost in the context of simplified template cross sections (STXS). These results are consistent with the expectation of a Higgs boson with a mass of $125 \mathrm{GeV}$ as well.

\section{Search for CP violation}

Events selected in the $H \rightarrow \tau \tau$ analysis can be used to examine the properties of the Higgs boson with respect to charge and parity conjugation (CP). This is particularly interesting for the potential to see a new source of $\mathrm{CP}$ violation in the Higgs sector.

There are two complementary approaches to measuring the CP properties of the Higgs boson. Both interpret the result in the context of effective field theory where the SM Lagrangian is extended 
with CP-odd operators. Parameters in the additional terms then determine the admixture of CP-odd contributions.

One approach is probing the $H V V$ vertex in the VBF production mode. This is done for VBF events selected in $20.3 \mathrm{fb}^{-1}$ of data collected at a centre-of-mass energy of $8 \mathrm{TeV}$ [7]. Only the $\tau_{\text {lep }} \tau_{\text {had }}$ and $\tau_{\text {had }} \tau_{\text {had }}$ categories are included. The observable used in this analysis which is sensitive to a CP-odd Higgs boson is the Optimal Observable. It is a ratio of Matrix Elements, where the numerator is the CP-odd interference term between CP-even and CP-odd parts and the denominator is the purely SM-like part (CP-even). The result is expressed in the $\tilde{d}$ parameter which is equal to zero for the SM (CP-even) hypothesis. The observed value lies in the interval $[-0.11,0.05]$ at the $68 \%$ confidence level, so is fully consistent with a CP-even Higgs boson.

The other approach to measure the CP properties is targeting the $H f f$ vertex of the decay. Prospects for measurements in the $H \rightarrow \tau \tau$ decay at the HL-LHC with $3000 \mathrm{fb}^{-1}$ at $14 \mathrm{TeV}$ are reported in [8]. The analysis strategy is to measure the difference in transverse spin correlations in the decay of a CP-even or CP-odd Higgs decay to tau lepton pairs. The tau lepton is the prime candidate for this as information of its spin direction is in turn propagated to the angular distributions of its visible decay products. Here the case of the decay $\tau^{ \pm} \rightarrow \rho^{ \pm} v_{\tau} \rightarrow \pi^{ \pm} \pi^{0} v_{\tau}$ is considered for both tau leptons. This is $6 \%$ of all $H \rightarrow \tau \tau$ events. The sensitive observable here is the acoplanarity angle $\varphi^{\star}$ between the planes spanned by the $\rho^{ \pm}$decay products in the $\rho^{+} \rho^{-}$rest-frame. For this definition it is necessary to reconstruct the 4-momenta of both the charged and the neutral pion in the tau lepton decay. The projected result is expected to be able to set confidence intervals at $68 \%$ $\mathrm{CL}$ on a CP-even/odd mixing angle $\phi_{\tau}$ with a precision between $\pm 18^{\circ}$ and $\pm 33^{\circ}$ depending on the assumed $\pi^{0}$ momentum resolution. Additionally even with a $\pi^{0}$ momentum resolution twice as bad as foreseen, the purely CP-odd hypothesis may be excluded with a significance of 2 standard deviations.

\section{Conclusion}

The existence of the $H \rightarrow \tau \tau$ process and thereby the corresponding Yukawa coupling is proven by ATLAS with high significance and the cross section at $13 \mathrm{TeV}$, measured in LHC protonproton collisions, is consistent with predictions for a SM Higgs boson of $125 \mathrm{GeV}$.

Furthermore, CP properties of the Higgs boson can be probed using the ditau decay channel. A measurement at $8 \mathrm{TeV}$ targeting the $H V V$ vertex in VBF production is consistent with a SM (CPeven) Higgs boson at a confidence level of $68 \%$. Future CP measurements in the ditau Higgs boson decay targeting the $H f f$ decay vertex at the HL-LHC have potential to achieve $95 \%$ confidence level.

\section{References}

[1] ATLAS Collaboration, The ATLAS Experiment at the CERN Large Hadron Collider, JINST 3 (2008) S08003.

[2] F. Englert and R. Brout, Broken Symmetry and the Mass of Gauge Vector Mesons, Phys. Rev. Lett. 13 (1964) 321. 
[3] P. W. Higgs, Broken Symmetries and the Masses of Gauge Bosons, Phys. Rev. Lett. 13 (1964) 508.

[4] G. S. Guralnik, C. R. Hagen, and T. W. B. Kibble, Global Conservation Laws and Massless Particles, Phys. Rev. Lett. 13 (1964) 585.

[5] S. Weinberg, A Model of Leptons, Phys. Rev. Lett. 19 (1967) 1264.

[6] ATLAS Collaboration, Cross-section measurements of the Higgs boson decaying into a pair of $\tau$-leptons in proton-proton collisions at $\sqrt{s}=13 \mathrm{TeV}$ with the ATLAS detector, Phys. Rev. D 99 (2019) 072001, arXiv: 1811.08856 [hep-ex] .

[7] ATLAS Collaboration, Test of CP Invariance in vector-boson fusion production of the Higgs boson using the Optimal Observable method in the ditau decay channel with the ATLAS detector, Eur. Phys. J. C 76 (2016) 658, arXiv: 1602.04516 [hep-ex] .

[8] ATLAS Collaboration,

Probing the $\mathscr{C} \mathscr{P}$ nature of the Higgs boson coupling to $\tau$ leptons at HL-LHC, ATL-PHYS-PUB-2019-008, 2019, URL: https://cds.cern.ch/record/2665667.

[9] ATLAS Collaboration, Measurement of the tau lepton reconstruction and identification performance in the ATLAS experiment using pp collisions at $\sqrt{s}=13 \mathrm{TeV}$, ATLAS-CONF-2017-029, 2017, URL: https: / / cds . cern. ch/record/2261772.

[10] A. Elagin, P. Murat, A. Pranko, and A. Safonov, A New Mass Reconstruction Technique for Resonances Decaying to $\tau \tau$, Nucl. Instrum. Meth. A 654 (2011) 481, arXiv: 1012.4686 [hep-ex] . 